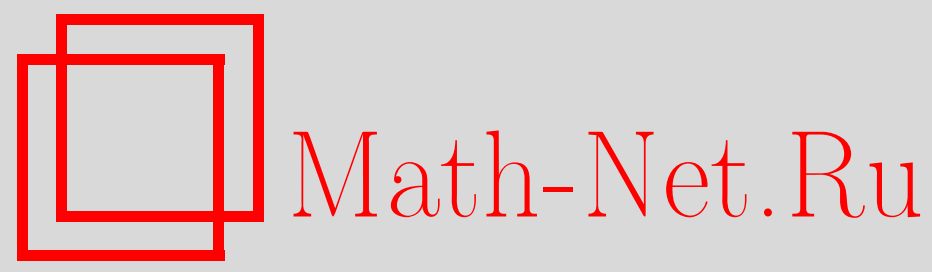

В. Г. Саргсян, О числе $k$-сумм в группах простого порядка, Дискрет. матем., 2012, том 24, выпуск 3, 25-38

DOI: https://doi.org/10.4213/dm1194

Использование Общероссийского математического портала Math-Net.Ru подразумевает, что вы прочитали и согласны с пользовательским соглашением http://www.mathnet.ru/rus/agreement

Параметры загрузки:

IP : 54.89 .56 .158

26 апреля 2023 г., 17:12:41 
УДК 519.1

\title{
О числе $k$-сумм в группах простого порядка
}

\author{
() 2012 г. $\quad$ В. Г. Саргсян
}

Подмножество $A$ элементов группы $\mathbf{G}$ называется $k$-суммой, если существует подмножество $B \subseteq \mathbf{G}$ такое, что $A=k B$, где

$$
k B=\left\{x_{1}+\ldots+x_{k}: x_{1}, \ldots, x_{k} \in B\right\} .
$$

Получены верхняя и нижняя оценки числа $k$-сумм в группах простого порядка.

Работа выполнена при поддержке РФФИ, проект 10-01-00768-а.

\section{1. Введение}

Пусть $\mathbf{Z}_{p}$ - группа вычетов по простому модулю $p$ и $k \geqslant 2-$ натуральное число. Для всякого $B \subseteq \mathbf{Z}_{p}$ и любого натурального числа $k$ положим

$$
k B=\left\{x_{1}+\ldots+x_{k} \quad(\bmod p): x_{1}, \ldots, x_{k} \in B\right\} .
$$

Подмножество $A \subseteq \mathbf{Z}_{p}$ называется $k$-суммой, если существует подмножество $B \subseteq \mathbf{Z}_{p}$ такое, что $A=k B$. Семейство всех $k$-сумм в группе $\mathbf{Z}_{p}$ обозначим через $\mathbf{S S}_{k}\left(\mathbf{Z}_{p}\right)$.

В 2004 г. Б. Грин и И. Ружа [1] доказали, что

$$
p^{2} 2^{p / 3} \ll\left|\mathbf{S S}_{2}\left(\mathbf{Z}_{p}\right)\right| \leqslant 2^{p / 3+\theta(p)},
$$

где $\theta(p) / p \rightarrow 0$ при $p \rightarrow \infty$, причем

$$
\theta(p) \ll p(\log \log p)^{2 / 3}(\log p)^{-1 / 9}
$$

(здесь и далее все логарифмы берутся по основанию два).

Целью настоящей работы является получение оценки числа $\left|\mathbf{S S}_{k}\left(\mathbf{Z}_{p}\right)\right|$.

Теорема 1. Пусть $p-$ простое число, а $k \geqslant 2$ - натуральное число. Тогда существует положительная константа $\mathbf{C}_{k}$ такая, что выполняются неравенства

$$
\mathbf{C}_{k} 2^{p /(2 k-1)} \leqslant\left|\mathbf{S S}_{k}\left(\mathbf{Z}_{p}\right)\right| \leqslant 2^{p /(k+1)+(k-2)+o(p)} .
$$

\section{2. Определения и вспомогательные утверждения}

Пусть $\mathbf{R}$ - множество действительных чисел, $f_{i}: \mathbf{Z}_{p} \rightarrow \mathbf{R}, i=1, \ldots, k$, и $x \in \mathbf{Z}_{p}$, тогда положим

$$
\left(f_{1} * \ldots * f_{k}\right)(x)=\sum_{x_{1} \in \mathbf{Z}_{p}} \ldots \sum_{x_{k-1} \in \mathbf{Z}_{p}} f_{1}\left(x_{1}\right) \cdots f_{k-1}\left(x_{k-1}\right) f_{k}\left(x-x_{1}-\ldots-x_{k-1}\right) .
$$


Пусть

$$
\hat{f}(x)=\sum_{y \in \mathbf{Z}_{p}} f(y) e^{2 \pi i x y / p},
$$

и для всякого $A \subseteq \mathbf{Z}_{p}$ и любого целого числа $d$ положим

$$
d \star A=\{d a \quad(\bmod p): a \in A\} .
$$

Функция $\hat{f}(x)$ называется преобразованием Фурье функции $f$, а $d \star A$ назовем растяжением множества $A$. Заметим, что для любого $x \in \mathbf{Z}_{p}$

$$
\left(\overline{f_{1} * \ldots * f_{k}}\right)(x)=\hat{f}_{1}(x) \cdots \hat{f}_{k}(x) \text {. }
$$

Действительно,

$$
\begin{aligned}
\left(\widehat{f_{1} * \ldots * f_{k}}\right)(x)= & \sum_{y \in \mathbf{Z}_{p}}\left(f_{1} * \ldots * f_{k}\right)(y) e^{2 \pi i y x / p} \\
= & \sum_{y \in \mathbf{Z}_{p}} \sum_{y_{1} \in \mathbf{Z}_{p}} \ldots \sum_{y_{k-1} \in \mathbf{Z}_{p}} f_{1}\left(y_{1}\right) \cdots f_{k-1}\left(y_{k-1}\right) \\
& \quad \times f_{k}\left(y-y_{1}-\ldots-y_{k-1}\right) e^{2 \pi i y_{1} x / p} \ldots e^{2 \pi i y_{k-1} x / p} \\
& \quad \times e^{2 \pi i\left(y-y_{1}-\ldots-y_{k-1}\right) x / p} \\
= & \sum_{y_{1} \in \mathbf{Z}_{p}} f_{1}\left(y_{1}\right) e^{2 \pi i y_{1} x / p} \ldots \sum_{y_{k-1} \in \mathbf{Z}_{p}} f_{k-1}\left(y_{k-1}\right) \\
& \quad \times e^{2 \pi i y_{k-1} x / p} \sum_{y \in \mathbf{Z}_{p}} f_{k}\left(y-y_{1}-\ldots-y_{k-1}\right) \\
= & \quad \hat{f}_{1}(x) \cdots e^{2 \pi i\left(y-y_{1}-\ldots-y_{k-1}\right) x / p}
\end{aligned}
$$

Пусть $A_{1}, \ldots, A_{k}$ - непустые подмножества группы $\mathbf{Z}_{p}$, а характеристические функции множеств $A_{1}, \ldots, A_{k}$ обозначим, соответственно, через $\chi_{A_{1}}(x), \ldots, \chi_{A_{k}}(x)$. Тогда $\left(\chi_{A_{1}} * \ldots * \chi_{A_{k}}\right)(x)$ - это количество наборов $\left(x_{1}, \ldots, x_{k}\right) \in A_{1} \times \ldots \times A_{k}$ таких, что $x \equiv x_{1}+\ldots+x_{k}(\bmod p)$. Положим

$$
\begin{aligned}
A_{1}+\ldots+A_{k} & =\left\{x_{1}+\ldots+x_{k} \quad(\bmod p): x_{1} \in A_{1}, \ldots, x_{k} \in A_{k}\right\}, \\
S_{h, k}\left(A_{1}, \ldots, A_{k}\right) & =\left\{x \in \mathbf{Z}_{p}:\left(\chi_{A_{1}} * \ldots * \chi_{A_{k}}\right)(x) \geqslant h\right\}, \quad h>0 .
\end{aligned}
$$

Теорема 2 ([2]). Пусть $A_{1}, \ldots, A_{k}-$ непустые подмножества группьы $\mathbf{Z}_{p}$. Тогда

$$
\left|A_{1}+\ldots+A_{k}\right| \geqslant \min \left(p,\left|A_{1}\right|+\ldots+\left|A_{k}\right|-(k-1)\right) .
$$

Теорема 3 ([3]). Пусть $A_{1}, A_{2}-$ непустые подмножества группьь $\mathbf{Z}_{p}$. Тогда

$$
\left|S_{1,2}\left(A_{1}, A_{2}\right)\right|+\ldots+\left|S_{t, 2}\left(A_{1}, A_{2}\right)\right| \geqslant t \min \left(p,\left|A_{1}\right|+\left|A_{2}\right|-t\right),
$$

где $t \leqslant \min \left(\left|A_{1}\right|,\left|A_{2}\right|\right)$.

Из теорем 2, 3 вытекают следующие два утверждения. 
Лемма 1. Пусть $A_{1}, \ldots, A_{k}-$ непустье подмножества группь $\mathbf{Z}_{p}$. Тогда

$$
\left|S_{1, k}\left(A_{1}, \ldots, A_{k}\right)\right|+\ldots+\left|S_{t, k}\left(A_{1}, \ldots, A_{k}\right)\right| \geqslant t \min \left(p,\left|A_{1}\right|+\ldots+\left|A_{k}\right|-t-k+2\right),
$$

где $t \leqslant \min \left(\left|A_{1}\right|, \ldots,\left|A_{k}\right|\right)$.

Доказательство. Предположим, что

$$
\left|A_{1}\right|=\min \left(\left|A_{1}\right|, \ldots,\left|A_{k}\right|\right) .
$$

В силу теоремы 3,

$$
\begin{aligned}
&\left|S_{1,2}\left(A_{1},\left(A_{2}+\ldots+A_{k}\right)\right)\right|+\ldots+\left|S_{t, 2}\left(A_{1},\left(A_{2}+\ldots+A_{k}\right)\right)\right| \\
& \geqslant t \min \left(p,\left|A_{1}\right|+\left|A_{2}+\ldots+A_{k}\right|-t\right),
\end{aligned}
$$

где $t \leqslant\left|A_{1}\right|$.

С другой стороны, в силу теоремы 2

$$
\left|A_{2}+\ldots+A_{k}\right| \geqslant \min \left(p,\left|A_{2}\right|+\ldots+\left|A_{k}\right|-(k-2)\right) .
$$

Подставив (4) в (3), получим, что

$$
\begin{aligned}
& \left|S_{1, k}\left(A_{1}, \ldots, A_{k}\right)\right|+\ldots+\left|S_{t, k}\left(A_{1}, \ldots, A_{k}\right)\right| \\
& \quad \geqslant\left|S_{1,2}\left(A_{1},\left(A_{2}+\ldots+A_{k}\right)\right)\right|+\ldots+\left|S_{t, 2}\left(A_{1},\left(A_{2}+\ldots+A_{k}\right)\right)\right| \\
& \quad \geqslant t \min \left(p,\left|A_{1}\right|+\ldots+\left|A_{k}\right|-t-k+2\right) .
\end{aligned}
$$

Лемма доказана.

Лемма 2. Пусть $A_{1}, \ldots, A_{k}-$ непустые подмножества группь $\mathbf{Z}_{p} u$

$$
h \leqslant \min \left(\left|A_{1}\right|, \ldots,\left|A_{k}\right|\right) \text {. }
$$

Тогда

$$
\left|S_{h, k}\left(A_{1}, \ldots, A_{k}\right)\right| \geqslant \min \left(p,\left|A_{1}\right|+\ldots+\left|A_{k}\right|-k+2\right)-2(h p)^{1 / 2} .
$$

Доказательство. Заметим, что при $i \leqslant j$ выполняется

$$
\left|S_{i, k}\left(A_{1}, \ldots, A_{k}\right)\right| \geqslant\left|S_{j, k}\left(A_{1}, \ldots, A_{k}\right)\right| .
$$

Тогда, если взять

$$
h \leqslant t \leqslant \min \left(\left|A_{1}\right|, \ldots,\left|A_{k}\right|\right),
$$

то в силу леммы 1 получим, что

$$
\begin{aligned}
t \min & \left(p,\left|A_{1}\right|+\ldots+\left|A_{k}\right|-t-k+2\right) \\
& \leqslant\left|S_{1, k}\left(A_{1}, \ldots, A_{k}\right)\right|+\ldots+\left|S_{t, k}\left(A_{1}, \ldots, A_{k}\right)\right| \\
& \leqslant h p+t\left|S_{h, k}\left(A_{1}, \ldots, A_{k}\right)\right| .
\end{aligned}
$$

Полагая $t=(h p)^{1 / 2}$, получим, что

$$
\begin{aligned}
\min & \left(p,\left|A_{1}\right|+\ldots+\left|A_{k}\right|-k+2\right)-2(h p)^{1 / 2} \\
& \leqslant \min \left(p,\left|A_{1}\right|+\ldots+\left|A_{k}\right|-k-(h p)^{1 / 2}+2\right)-(h p)^{1 / 2} \\
& \leqslant\left|S_{h, k}\left(A_{1}, \ldots, A_{k}\right)\right| .
\end{aligned}
$$

Лемма доказана. 
Лемма 3. Пусть

$$
\mathbf{T}_{k, s}\left(\mathbf{Z}_{p}\right)=\left\{A \subset \mathbf{Z}_{p}:|A| \leqslant p /(k+1) s\right\} .
$$

Тогда существует s такое, что

$$
\left|\mathbf{T}_{k, s}\left(\mathbf{Z}_{p}\right)\right| \leqslant 2^{p /(k+1)} .
$$

Доказательство. Пусть $n, m-$ целые числа, $1 \leqslant m \leqslant n$. Тогда (см. лемму 6.8 в [4])

$$
\sum_{0 \leqslant i \leqslant m}\left(\begin{array}{l}
n \\
i
\end{array}\right) \leqslant\left(\frac{e n}{m}\right)^{m} .
$$

Выберем $s$, удовлетворяющее условию

$$
e s(k+1) \leqslant 2^{s} .
$$

Тогда из (6) получаем, так как $n=p$ и $m=p /(k+1) s$, что

$$
\begin{aligned}
\left|\mathbf{T}_{k, s}\left(\mathbf{Z}_{p}\right)\right| & =\sum_{0 \leqslant i \leqslant p /(k+1) s}\left(\begin{array}{l}
p \\
i
\end{array}\right) \leqslant(e s(k+1))^{p /(k+1) s} \\
& \leqslant\left(2^{s}\right)^{p /(k+1) s}=2^{p /(k+1)} .
\end{aligned}
$$

Лемма доказана.

Пусть $L$ - натуральное число. Для каждого $y \in\{0, \ldots, p-1\}$ определим разбиение $\mathbf{R}_{y, L}$ группы $\mathbf{Z}_{p}$ на интервалы вида

$$
J_{i}^{y}=\{(i L+1+y) \quad(\bmod p), \ldots,((i+1) L+y) \quad(\bmod p)\},
$$

где $0 \leqslant i \leqslant\lfloor p / L\rfloor-1$. Все интервалы $J_{i}^{y}$ из $\mathbf{R}_{y, L}$ имеют длину $L$, а множество

$$
J_{y}=\mathbf{Z}_{p} \backslash \bigcup_{i} J_{i}^{y}
$$

имеет мощность $p-L\lfloor p / L\rfloor<L$. Множество $A \subseteq \mathbf{Z}_{p}$ называется $L$-гранулированным (см. [1]), если для некоторого целого числа $d<p$ и некоторого разбиения $\mathbf{R}_{y, L}$ растяжение $d \star A$ является объединением нескольких интервалов $J_{i}^{y}$ разбиения $\mathbf{R}_{y, L}$, отличных от $J_{y}$. Обозначим множество $L$-гранулированных подмножеств группы $\mathbf{Z}_{p}$ через $\mathbf{G}_{L}\left(\mathbf{Z}_{p}\right)$.

Лемма 4. Справедливо неравенство

$$
\left|\mathbf{G}_{L}\left(\mathbf{Z}_{p}\right)\right| \leqslant p 2^{p / L} .
$$

Доказательство. Число подмножеств гранул (интервалов) разбиения $R_{y, L}$ группы $\mathbf{Z}_{p}$ обозначим через $g\left(R_{y, L}\right)$, а число различных разбиений $R_{y, L}-$ через $r(L)$. Очевидно, что

$$
\left|\mathbf{G}_{L}\left(\mathbf{Z}_{p}\right)\right| \leqslant g\left(R_{y, L}\right) r(L) .
$$

Заметим, что число гранул разбиения $\mathbf{R}_{y, L}$ группы $\mathbf{Z}_{p}$ равно $\lfloor p / L\rfloor$, а количество различных разбиений $\mathbf{R}_{y, L}$ группы $\mathbf{Z}_{p}$ равно $p$. Отсюда и из неравенства (9) вытекает справедливость неравенства (8). Лемма доказана. 
Лемма 5. Пусть $A \subseteq \mathbf{Z}_{p} u|A|=\alpha p$, и пусть $\varepsilon_{1}, \varepsilon_{2}, \varepsilon_{3}-$ положительные действительные числа, $k \geqslant 2$ и L - натуральные числа, и $p$ - простое число, такое, что имеет место неравенство

$$
p>(\sqrt{8 k} L)^{4^{2 k} \alpha^{2(k-1)} \varepsilon_{1}^{-2 k} \varepsilon_{2}^{-2} \varepsilon_{3}^{-1} .}
$$

Тогда существует подмножество $A^{\prime} \subseteq \mathbf{Z}_{p}$ со следующими свойствами:

(i) $A^{\prime}$ является L-гранулированным;

(ii) $\left|A \backslash A^{\prime}\right| \leqslant \varepsilon_{1} p$;

(iii) множество $k A$ содержит все элементы $x \in \mathbf{Z}_{p}$, для которых

$$
(\underbrace{\chi_{A^{\prime}} * \ldots * \chi_{A^{\prime}}}_{k})(x) \geqslant \varepsilon_{2} p^{k-1},
$$

за возможным исключением не более $\varepsilon_{3}$ р элементов.

Доказательство. Пусть $h \in\{0, \ldots, p-1\}$ и $\mathbf{R}_{h, L}-$ разбиение группы $\mathbf{Z}_{p}$.

Докажем часть (i). Множество $A^{\prime}$ определим как объединение интервалов $J_{i}^{h}$ разбиения $\mathbf{R}_{h, L}$ таких, что

$$
\left|A \cap J_{i}^{h}\right| \geqslant \frac{\varepsilon_{1} L}{2} .
$$

По определению очевидно, что $A^{\prime} L$-гранулировано.

Докажем часть (ii). Пусть $x \in A \backslash A^{\prime}$, тогда либо $x \in J_{h}$, либо $x \in A \cap J_{i}^{h}, i=$ $0, \ldots,\lfloor p / L\rfloor-1$, и $\left|A \cap J_{i}^{h}\right| \leqslant \varepsilon_{1} L / 2$. В первом случае имеем $\left|J_{h}\right|<L$, а из неравенства (10) следует, что $L \leqslant \varepsilon_{1} p / 2$. Итак, получаем

$$
\left|A \backslash A^{\prime}\right| \leqslant \frac{\varepsilon_{1} L}{2} \frac{p}{L}+L \leqslant \varepsilon_{1} p .
$$

Докажем часть (iii). Пусть $x \in \mathbf{Z}_{p}$ и $\hat{\chi}_{A}(x)$ - преобразование Фурье характеристической функции $\chi_{A}$ множества $A$. Тогда

$$
\hat{\chi}_{A}(x)=\sum_{y \in \mathbf{Z}_{p}} \chi_{A}(y) e^{2 \pi i y x / p}=\sum_{y \in A} e^{2 \pi i y x / p} .
$$

Положим

$$
\delta=4^{-k} \varepsilon_{1}^{k} \varepsilon_{2} \varepsilon_{3}^{1 / 2} \alpha^{-k+3 / 2},
$$

где $\varepsilon_{1}, \varepsilon_{2}$ и $\varepsilon_{3}$ взяты из неравенства (10). Положим

$$
\mathbf{D}=\left\{x \neq 0:\left|\hat{\chi}_{A}(x)\right| \geqslant \delta p\right\} .
$$

Определим функцию $f(x)$ следующим образом:

$$
f(x)=\frac{1}{2 L-1} \sum_{j=-(L-1)}^{L-1} e^{2 \pi i j q x / p} .
$$


Для доказательства части (iii) необходимо показать, что существует $q \in \mathbf{Z}_{p} \backslash\{0\}$ такое, что для всех $x \in \mathbf{Z}_{p}$ выполняется неравенство

$$
\left|\hat{\chi}_{A}(x)\right|\left|1-f^{k}(x)\right| \leqslant \delta p .
$$

Неравенство (11), очевидно, имеет место для $x=0$, так как $f(0)=1$, а также в случае, когда $\left|\hat{\chi}_{A}(x)\right| \leqslant \delta p$, поскольку $f(x) \in[-1,1]$. Итак, осталось доказать существование такого $q$, что неравенство (11) выполнено для всех $x \in \mathbf{D}$. Оценим величину $1-f(x)$. Обозначим через $\langle x\rangle$ расстояние от $x$ до ближайшего целого числа. Воспользуемся тем, что

$$
1-\cos (2 \pi x) \leqslant 2 \pi^{2}\langle x\rangle^{2}
$$

Нетрудно видеть, что

$$
\begin{aligned}
1-f(x) & =\frac{2}{2 L-1} \sum_{j=1}^{L-1}\left(1-\cos \frac{2 \pi j q x}{p}\right) \leqslant \frac{4 \pi^{2}}{2 L-1} \sum_{j=1}^{L-1}\left\langle\frac{j q x}{p}\right\rangle^{2} \\
& \leqslant \frac{4 \pi^{2}}{2 L-1}\left\langle\frac{q x}{p}\right\rangle^{2} \sum_{j=1}^{L-1} j^{2} \leqslant \frac{2 \pi^{2} L^{2}}{3}\left\langle\frac{q x}{p}\right\rangle^{2} .
\end{aligned}
$$

Воспользуемся тем, что при условии $|x| \leqslant 1$

$$
1-x^{k}=(1-x)\left(1+x+x^{2}+\ldots+x^{k-1}\right) \leqslant k(1-x) .
$$

Из (12) и (13) следует, что

$$
\left|\hat{\chi}_{A}(x)\right|\left|1-f^{k}(x)\right| \leqslant k\left|\hat{\chi}_{A}(x)\right||1-f(x)| \leqslant 8 k L^{2}\langle q x / p\rangle^{2}\left|\hat{\chi}_{A}(x)\right| .
$$

Заметим, что при выполнении неравенства

$$
\left\langle\frac{q x}{p}\right\rangle \leqslant \frac{1}{\sqrt{8 k} L}\left(\frac{\delta p}{\left|\hat{\chi}_{A}(x)\right|}\right)^{1 / 2}
$$

для некоторого $q \in \mathbf{Z}_{p} \backslash\{0\}$ и для всех $x \in \mathbf{D}$ неравенство (11) также выполняется.

Докажем, что такое $q$ действительно существует. По определению,

$$
\langle q x / p\rangle=\min \{(q x \quad(\bmod p)) / p,(p-q x \quad(\bmod p)) / p\} .
$$

Положим

$$
d=|\mathbf{D}|, \quad \mathbf{D}=\left\{r_{1}, \ldots, r_{d}\right\},
$$

и

$$
a_{i}=\frac{1}{\sqrt{8 k} L}\left(\frac{\delta p}{\left|\hat{\chi}_{A}\left(r_{i}\right)\right|}\right)^{1 / 2} .
$$

Тогда неравенство (14) можно переписать в виде

$$
\min \left\{q r_{i} \quad(\bmod p), p-q r_{i} \quad(\bmod p)\right\} \leqslant p a_{i}, \quad i=1, \ldots, d .
$$


Обозначим

$$
\mathbf{Z}_{p}^{d}=\left\{\left(x_{1}, \ldots, x_{d}\right): x_{1}, \ldots, x_{d} \in \mathbf{Z}_{p}\right\}
$$

Разобьем $\mathbf{Z}_{p}^{d}$ на непересекающиеся подмножества

$$
\mathbf{Z}_{p}^{d}=\bigcup_{\left(i_{1}, \ldots, i_{d}\right)} \mathbf{Q}_{i_{1}, \ldots, i_{d}}
$$

где

$$
\mathbf{Q}_{i_{1}, \ldots, i_{d}}=\left\{\left(x_{1}, \ldots, x_{d}\right): i_{j} p a_{j}<x_{j} \leqslant\left(i_{j}+1\right) p a_{j}, j=1, \ldots, d\right\} .
$$

Пусть $\mu_{d}$ - количество различных множеств $\mathbf{Q}_{i_{1}, \ldots, i_{d}}$. С учетом того, что $0 \leqslant i_{j} \leqslant 1 / a_{j}-1$, $j=1, \ldots, d$, получаем, что

$$
\mu_{d} \leqslant \prod_{i=1}^{d} \frac{1}{a_{i}}
$$

Рассмотрим следующие $p-1$ элементов из $\mathbf{Z}_{p}^{d}:\left(q r_{1}(\bmod p), \ldots, q r_{d}(\bmod p)\right)$, где $r_{1}, \ldots, r_{d} \in \mathbf{D}, q=1, \ldots, p-1$. Покажем, что если

$$
p>\prod_{i=1}^{d} \frac{1}{a_{i}}=(\sqrt{8 k} L)^{d}\left(\prod_{x \in \mathbf{D}} \frac{\left|\hat{\chi}_{A}(x)\right|}{\delta p}\right)^{1 / 2} \geqslant \mu_{d},
$$

то существует $q$ такое, что для всех $r_{i} \in \mathbf{D}, i=1, \ldots, d$, выполняется неравенство (15). Рассмотрим два случая.

(А) Пусть $\mu_{d}=p-1$, тогда в качестве $q$ выберем $q_{0} \in \mathbf{Z}_{p} \backslash\{0\}$ такое, что $\left(q_{0} r_{1}(\bmod p), \ldots, q_{0} r_{d}(\bmod p)\right) \in \mathbf{Q}_{0, \ldots, 0}$. Нетрудно заметить, что при $q=q_{0}$ неравенство (15) выполняется для всех $r_{i} \in \mathbf{D}, i=1, \ldots, d$.

(В) Пусть $\mu_{d}<p-1$, тогда по принципу Дирихле существуют $q_{1}, q_{2} \in \mathbf{Z}_{p} \backslash\{0\}$ такие, что векторы $\left(q_{1} r_{1}(\bmod p), \ldots, q_{1} r_{d}(\bmod p)\right)$ и $\left(q_{2} r_{1}(\bmod p), \ldots, q_{2} r_{d}(\bmod p)\right)$ принадлежат одному и тому же множеству из $\mathbf{Q}_{i_{1}, \ldots, i_{d}}$. Очевидно, что при $q=q_{1}-q_{2}$ неравенство (15) выполняется для всех $r_{i} \in \mathbf{D}, i=1, \ldots, d$.

Теперь покажем, что неравенство (16) является следствием условия (10). Действительно, в силу равенства Парсеваля

$$
p^{-1}\left(\sum_{x \in \mathbf{D}}\left|\hat{\chi}_{A}(x)\right|^{2}+\sum_{x \in \mathbf{Z}_{p} \backslash \mathbf{D}}\left|\hat{\chi}_{A}(x)\right|^{2}\right)=\sum_{x \in \mathbf{Z}_{p}}\left|\chi_{A}(x)\right|^{2}=\alpha p .
$$

Из (17) следует, что

$$
\sum_{x \in \mathbf{D}}\left|\hat{\chi}_{A}(x)\right|^{2} \leqslant \alpha p^{2}
$$

Из неравенства (18) и неравенства о среднем арифметическом и среднем геометрическом следует, что

$$
\left(\prod_{x \in \mathbf{D}}\left|\hat{\chi}_{A}(x)\right|^{2}\right)^{1 / d} \leqslant \frac{1}{d} \sum_{x \in \mathbf{D}}\left|\hat{\chi}_{A}(x)\right|^{2} \leqslant \frac{\alpha p^{2}}{d}
$$


Отсюда следует, что

$$
\prod_{x \in \mathbf{D}}\left|\hat{\chi}_{A}(x)\right| \leqslant\left(\frac{\alpha p^{2}}{d}\right)^{d / 2}
$$

Из (19) получаем, что

$$
(\sqrt{8 k} L)^{d}\left(\prod_{x \in \mathbf{D}} \frac{\left|\hat{\chi}_{A}(x)\right|}{\delta p}\right)^{1 / 2} \leqslant\left(\sqrt{8 k} L \alpha^{1 / 4} \delta^{-1 / 2} d^{-1 / 4}\right)^{d} .
$$

Нетрудно заметить, что правая часть неравенства (20) является возрастающей функцией от $d$ при $d<64 k^{2} L^{4} \alpha / \delta^{2}$ ею С другой стороны, из (18) имеем $d \delta^{2} p^{2} \leqslant \alpha p^{2}$, а значит, $d \leqslant \alpha / \delta^{2}$. Следовательно,

$$
\left(\sqrt{8 k} L \alpha^{1 / 4} \delta^{-1 / 2} d^{-1 / 4}\right)^{d} \leqslant(\sqrt{8 k} L)^{\alpha / \delta^{2}} .
$$

Полагая

$$
\delta=4^{-k} \varepsilon_{1}^{k} \varepsilon_{2} \varepsilon_{3}^{1 / 2} \alpha^{-k+3 / 2},
$$

получаем, что существует $q$ такое, что неравенство (11) выполняется. При этом без ограничения общности можно считать $q=1$ (этого можно добиться выбором соответствующего растяжения множества $A)$.

Определим функцию $\chi_{1}(x)$ следующим образом:

$$
\chi_{1}(x)=\frac{1}{|P|}\left(\chi_{A} * \chi_{P}\right)(x)
$$

где

$$
P=\{-(L-1), \ldots, L-1\}
$$

Из определения свертки следует, что

$$
\chi_{1}(x)=\frac{1}{|P|}|A \cap(P+x)|,
$$

а из равенства (2) получаем, что

$$
\hat{\chi}_{1}(x)=\hat{\chi}_{A}(x) f(x) .
$$

Отсюда, из равенства Парсеваля и равенства (2) следует, что

$$
\begin{aligned}
\sum_{x \in \mathbf{Z}_{p}} & \left|(\underbrace{\chi_{A} * \ldots * \chi_{A}}_{k})(x)-(\underbrace{\chi_{1} * \ldots * \chi_{1}}_{k})(x)\right|^{2} \\
& =p^{-1} \sum_{x \in \mathbf{Z}_{p}}\left|(\underbrace{\overline{\chi_{A} * \ldots * \chi_{A}}}_{k})(x)-(\underbrace{(\underbrace{}_{1 * \ldots * \chi_{1}}}_{k})(x)\right|^{2} \\
& =p^{-1} \sum_{x \in \mathbf{Z}_{p}}\left|\hat{\chi}_{A}^{k}(x)-\hat{\chi}_{1}^{k}(x)\right|^{2}=p^{-1} \sum_{x \in \mathbf{Z}_{p}}\left|\hat{\chi}_{A}(x)\right|^{2 k}\left|1-f^{k}(x)\right|^{2} \\
& \leqslant p^{-1}\left(\sup _{x \in \mathbf{Z}_{p}}\left|\hat{\chi}_{A}(x)\right|^{k-1}\left|1-f^{k}(x)\right|\right)^{2} \sum_{x \in \mathbf{Z}_{p}}\left|\hat{\chi}_{A}(x)\right|^{2} .
\end{aligned}
$$


Из определения следует, что

$$
\left|\hat{\chi}_{A}(x)\right|=\left|\sum_{y \in \mathbf{Z}_{p}} \chi_{A}(y) e^{2 \pi i y x / p}\right|=\left|\sum_{y \in A} e^{2 \pi i y x / p}\right| \leqslant \sum_{y \in A}\left|e^{2 \pi i y x / p}\right|=\alpha p .
$$

Из (11), (17), (22) и (23) получаем, что

$$
\begin{aligned}
\sum_{x \in \mathbf{Z}_{p}} \mid(\underbrace{\chi_{A} * \ldots * \chi_{A}}_{k})(x) & -\left.(\underbrace{\chi_{1} * \ldots * \chi_{1}}_{k})(x)\right|^{2} \\
& \leqslant\left(\sup _{x \in \mathbf{Z}_{p}}\left|\hat{\chi}_{A}(x)\right|\left|1-f^{k}(x)\right|\right)^{2} \alpha^{2 k-3} p^{2 k-3} \leqslant \alpha^{2 k-3} \delta^{2} p^{2 k-1} .
\end{aligned}
$$

Если $x \in A^{\prime}$, то существует интервал длины $L$, который содержит $x$ и, следовательно, содержится в интервале $[x-(L-1), \ldots, x+(L-1)]$. Из определения множества $A^{\prime}$ следует, что этот интервал содержит не менее $\varepsilon_{1} L / 2$ точек из $A$, а из определения функции $\chi_{1}(x)$ следует, что

$$
\chi_{1}(x) \geqslant \varepsilon_{1} / 4 \text {. }
$$

Заметим, что для всех $x \in \mathbf{Z}_{p}$ справедливо неравенство

$$
\chi_{1}(x) \geqslant \varepsilon_{1} \chi_{A^{\prime}}(x) / 4
$$

Из этого неравенства и определения свертки вытекает, что для всех $x \in \mathbf{Z}_{p}$

$$
(\underbrace{\chi_{1} * \ldots * \chi_{1}}_{k})(x) \geqslant \varepsilon_{1}^{k}(\underbrace{\chi_{A^{\prime} * \ldots * \chi_{A^{\prime}}}}_{k})(x) / 4^{k} .
$$

При условии, что

$$
(\underbrace{\chi_{A^{\prime}} * \ldots * \chi_{A^{\prime}}}_{k})(x) \geqslant \varepsilon_{2} p^{k-1},
$$

из (25) получаем, что

$$
(\underbrace{\chi_{1} * \ldots * \chi_{1}}_{k})(x) \geqslant \varepsilon_{1}^{k} \varepsilon_{2} p^{k-1} / 4^{k} .
$$

Покажем, что количество элементов $x \in \mathbf{Z}_{p}$ таких, что

$$
(\underbrace{\chi_{A} * \ldots * \chi_{A}}_{k})(x)=0
$$

и удовлетворяющих неравенству (26), не превосходит $\varepsilon_{3} p$. Семейство таких элементов обозначим через F. Заметим, что для всякого $x \in \mathbf{F}$

$$
\left|(\underbrace{\chi_{A} * \ldots * \chi_{A}}_{k})(x)-(\underbrace{\chi_{1} * \ldots * \chi_{1}}_{k})(x)\right|^{2} \geqslant \frac{\varepsilon_{1}^{2 k} \varepsilon_{2}^{2} p^{2(k-1)}}{4^{2 k}} .
$$


Из (24) и (28) получаем, что

$$
\begin{aligned}
\alpha^{2 k-3} \delta^{2} p^{2 k-1} & \geqslant \sum_{x \in \mathbf{Z}_{p}}\left|(\underbrace{\chi_{A} * \ldots * \chi_{A}}_{k})(x)-(\underbrace{\chi_{1} * \ldots * \chi_{1}}_{k})(x)\right|^{2} \\
& \geqslant \sum_{x \in \mathbf{F}}\left|(\underbrace{\chi_{A} * \ldots * \chi_{A}}_{k})(x)-(\underbrace{\chi_{1} * \ldots * \chi_{1}}_{k})(x)\right|^{2} \\
& \geqslant|\mathbf{F}| \frac{\varepsilon_{1}{ }^{2 k} \varepsilon_{2}^{2} p^{2(k-1)}}{4^{2 k}} .
\end{aligned}
$$

Отсюда следует, что

$$
|\mathbf{F}| \leqslant \frac{4^{2 k} \alpha^{2 k-3} \delta^{2}}{\varepsilon_{1}^{2 k} \varepsilon_{2}^{2}} p \leqslant \varepsilon_{3} p .
$$

Лемма доказана.

\section{3. Доказательство теоремы 1}

\section{1. Верхняя оценка}

Пусть $k \geqslant 2-$ натуральное число, а $s$ удовлетворяет условию (7). Рассмотрим разбиение множества $\mathbf{S S}_{k}\left(\mathbf{Z}_{p}\right)$ на две части:

$$
\mathbf{S S}_{k}\left(\mathbf{Z}_{p}\right)=\mathbf{S S}_{k, s}^{\prime}\left(\mathbf{Z}_{p}\right) \cup \mathbf{S S}_{k, s}^{\prime \prime}\left(\mathbf{Z}_{p}\right)
$$

где

$$
\begin{aligned}
& \mathbf{S S}_{k, s}^{\prime}\left(\mathbf{Z}_{p}\right)=\left\{B \in \mathbf{S S}_{k}\left(\mathbf{Z}_{p}\right): B=k A \text { и }|A| \leqslant p /(k+1) s\right\}, \\
& \mathbf{S S}_{k, s}^{\prime \prime}\left(\mathbf{Z}_{p}\right)=\left\{B \in \mathbf{S S}_{k}\left(\mathbf{Z}_{p}\right): B=k A \text { и }|A|>p /(k+1) s\right\} .
\end{aligned}
$$

Отсюда следует, что

$$
\left|\mathbf{S S}_{k}\left(\mathbf{Z}_{p}\right)\right| \leqslant\left|\mathbf{S S}_{k, s}^{\prime}\left(\mathbf{Z}_{p}\right)\right|+\left|\mathbf{S S}_{k, s}^{\prime \prime}\left(\mathbf{Z}_{p}\right)\right|
$$

С учетом того, что каждое множество $A \subseteq \mathbf{Z}_{p}$ порождает ровно одно множество вида $k A$, получаем, что

$$
\left|\mathbf{S S}_{k, s}^{\prime}\left(\mathbf{Z}_{p}\right)\right| \leqslant\left|\mathbf{T}_{k, s}\left(\mathbf{Z}_{p}\right)\right|
$$

В силу леммы (3) и неравенства (31),

$$
\left|\mathbf{S S}_{k, s}^{\prime}\left(\mathbf{Z}_{p}\right)\right| \leqslant 2^{p /(k+1)} .
$$

Теперь оценим сверху $\left|\mathbf{S S}_{k, s}^{\prime \prime}\left(\mathbf{Z}_{p}\right)\right|$. Пусть мощность множества $A \subseteq \mathbf{Z}_{p}$ превосходит $p /(k+1) s$ и $p-$ простое число, такое, что для натуральных чисел $k, L$ и положительных действительных чисел $\varepsilon_{1}, \varepsilon_{2}$ и $\varepsilon_{3}$ выполняется условие (10). Тогда, по лемме 5 , существует подмножество $A^{\prime}$, обладающее свойствами (i)-(iii). Оценим $\left|\mathbf{S S}_{k, s}^{\prime \prime}\left(\mathbf{Z}_{p}\right)\right|$ путем подсчета количества пар $\left(A^{\prime}, k A\right)$.

Пусть выбрано $A^{\prime} \in \mathbf{G}_{L}\left(\mathbf{Z}_{p}\right)$. Через $\bar{C}$ обозначим дополнение подмножества $C$ в $\mathbf{Z}_{p}$. 
В силу утверждения (iii) леммы 5 получаем, что $\overline{k A}$ является подмножеством объединения множества $\overline{S_{\varepsilon_{2} p^{k-1}, k}\left(\chi_{A^{\prime}}, \ldots, \chi_{A^{\prime}}\right)}$ и некоторого множества мощности, не превышающей $\varepsilon_{3} p$. В силу леммы 2 ,

$$
\left|S_{\varepsilon_{2} p^{k-1, k}}\left(\chi_{A^{\prime}}, \ldots, \chi_{A^{\prime}}\right)\right| \geqslant \min \left(p, k\left|A^{\prime}\right|-k+2\right)-2\left(\varepsilon_{2} p^{k}\right)^{1 / 2} .
$$

При условии $\left|A^{\prime}\right| \geqslant p /(k+1)$ получаем

$$
\begin{aligned}
\left|\overline{S_{\varepsilon_{2} p^{k-1}, k}\left(\chi_{A^{\prime}}, \ldots, \chi_{A^{\prime}}\right)}\right| & =p-\left|S_{\varepsilon_{2} p^{k-1}, k}\left(\chi_{A^{\prime}}, \ldots, \chi_{A^{\prime}}\right)\right| \\
& \leqslant p /(k+1)+2 \varepsilon_{2}{ }^{1 / 2} p^{k / 2}+(k-2) .
\end{aligned}
$$

Очевидно, что для любого подмножества $C \subseteq \mathbf{Z}_{p}$ множество $k C$ однозначно определяет множество $\overline{k C}$. Отсюда следует, что число способов выбора $k A$ при заданном $A^{\prime}$ мощности, превышающей $p /(k+1)$, не превосходит

$$
2^{p /(k+1)+(k-2)+\left(2 \varepsilon_{2}{ }^{1 / 2} p^{(k-2) / 2}+\varepsilon_{3}\right) p} .
$$

В силу утверждения (ii) леммы 5,

$$
|A| \leqslant\left|A^{\prime}\right|+\varepsilon_{1} p .
$$

При условии $\left|A^{\prime}\right|<p /(k+1)$ получаем, что

$$
|A|<p /(k+1)+\varepsilon_{1} p .
$$

С учетом того, что каждое множество $A \subseteq \mathbf{Z}_{p}$ порождает ровно одно множество вида $k A$, получаем, что число способов выбора $k A$ при заданном $A^{\prime}$ мощности, не превышающей $p /(k+1)$, не превосходит

$$
2^{p /(k+1)+\varepsilon_{1} p}
$$

В силу (33), (34), леммы 4 и леммы 5 с параметрами

$$
\varepsilon_{1}=\varepsilon_{3}=\varepsilon, \quad \varepsilon_{2}=\varepsilon^{2} p^{2-k}, \quad L=1+\lfloor 1 / \varepsilon\rfloor,
$$

получаем, что

$$
\left|\mathbf{S S}_{k, s}^{\prime \prime}\left(\mathbf{Z}_{p}\right)\right| \leqslant 2^{p /(k+1)+(k-2)+o(p)} .
$$

Из неравенств (30), (32) и (35) следует, что

$$
\left|\mathbf{S S}_{k}\left(\mathbf{Z}_{p}\right)\right| \leqslant 2^{p /(k+1)}+2^{p /(k+1)+(k-2)+o(p)}=2^{p /(k+1)+(k-2)+o(p)} .
$$

Верхняя оценка теоремы 1 доказана.

\section{2. Нижняя оценка}

Обозначим

$$
\mathbf{S S}_{k}\left(\mathbf{Z}_{p}, B\right)=\left\{A: A \in \mathbf{S S}_{k}\left(\mathbf{Z}_{p}\right) \text { и } B \subseteq A\right\} .
$$


Лемма 6. Пусть $k \geqslant 2$ - натуральное число, а $P \subseteq \mathbf{Z}_{p}-$ произвольная арифметическая прогрессия длины $k(\lfloor p /(2 k-1)\rfloor-1)-(k-1)$. Тогда существует положстельная константа $\mathbf{C}_{k}$ такая, что

$$
\left|\mathbf{S S}_{k}\left(\mathbf{Z}_{p}, P\right)\right| \geqslant \mathbf{C}_{k} 2^{p /(2 k-1)} .
$$

Доказательство. Положим

$$
L=\lfloor p /(2 k-1)\rfloor-1
$$

Без ограничения общности можно считать, что $P=\{k, \ldots, k L\}$. Пусть $B \subseteq\{1, \ldots, L\}$. Определим множество $A=A(B)$ равенством

$$
A=k(B \cup\{2 L+1\})=\bigcup_{i=0}^{k}\{(k-i) B+i(2 L+1)\} .
$$

С учетом того, что

$$
(B+(k-1)(2 L+1)) \cap\left(\bigcup_{i=0}^{k-2}\{(k-i) B+i(2 L+1)\}\right)=\varnothing,
$$

получаем, что различные $B \subseteq\{1, \ldots, L\}$ порождают различные множества $A$. Положим

$$
N(k)=\left\lceil\frac{\log \left(8 k^{2}\right)}{\log (4 / 3)}\right\rceil
$$

и пусть

$$
X=\{1, \ldots, N(k)\} \cup \bigcup_{i=1}^{k-1}(\lfloor(i+1) L / k\rfloor-N(k), \ldots,\lceil(i+1) L / k\rceil) .
$$

Множество $B \subseteq\{1, \ldots, L\}$ определим следующим образом:

$$
B=B(C)=X \cup C,
$$

где элементы множества $C$ выбраны из $\{1, \ldots, L\} \backslash X$ случайно с вероятностью $1 / 2$, независимо друг от друга. Положим

$$
Y=\{k, \ldots, k N(k)\} \cup \bigcup_{i=1}^{k-1}\{(i+1) L-k N(k), \ldots,(i+1) L\} .
$$

Очевидно, что $Y \subseteq k B$. Заметим, что если $x \notin k B$, то в представлении $x$ в виде $x=$ $x_{1}+\ldots+x_{k}$ существует как минимум одно $x_{i}, i \in\{1, \ldots, k\}$, такое, что $x_{i} \notin B$. Обозначим множество

$$
\mathbf{Q}_{k, L}(x)=\left\{\left(x_{1}, \ldots, x_{k}\right): x_{1}+\ldots+x_{k}=x, x_{1}, \ldots, x_{k} \in\{1, \ldots, L\}\right\} .
$$

Положим

$$
q=\left|\mathbf{Q}_{k, L}(x)\right|
$$


Будем говорить, что векторы $\left(x_{1}, \ldots, x_{k}\right)$ и $\left(y_{1}, \ldots, y_{k}\right)$ не пересекаются, если $\left\{x_{1}, \ldots, x_{k}\right\} \cap\left\{y_{1}, \ldots, y_{k}\right\}=\varnothing$.

Покажем, что для любого $x \in\{k N(k)+1, \ldots, L\}$ верно

$$
\mathbf{P}(x \notin k B) \leqslant\left(\frac{3}{4}\right)^{\lfloor x / k\rfloor} .
$$

С учетом того, что

$$
\mathbf{P}(A \& B) \leqslant \mathbf{P}(A)
$$

получаем, что

$$
\begin{aligned}
\mathbf{P}(x \notin k B) \leqslant & \mathbf{P}\left(\left(x_{1}^{1}+\ldots+x_{k}^{1} \notin k B\right) \& \ldots \&\left(x_{1}^{q}+\ldots+x_{k}^{q} \notin k B\right):\right. \\
& \left.\left(x_{1}^{i}, \ldots, x_{k}^{i}\right) \in \mathbf{Q}_{k, L}(x), i=1, \ldots, q\right) \\
\leqslant & \mathbf{P}\left(\left(x_{1}^{11}+\ldots+x_{k}^{11} \notin k B\right) \& \ldots \&\left(x_{1}^{1 n}+\ldots+x_{k}^{1 n} \notin k B\right)\right) \\
= & \mathbf{P}\left(\left(x_{1}^{11} \notin B \vee \ldots \vee x_{k}^{11} \notin B\right) \& \ldots \&\left(x_{1}^{1 n} \notin B \vee \ldots \vee x_{k}^{1 n} \notin B\right)\right),
\end{aligned}
$$

где векторы $\left(x_{1}^{11}, \ldots, x_{k}^{11}\right), \ldots,\left(x_{1}^{1 n}, \ldots, x_{k}^{1 n}\right), n \leqslant q$, попарно не пересекаются. Отсюда следует, что

$$
\begin{aligned}
& \mathbf{P}\left(\left(x_{1}^{11} \notin B \vee \ldots \vee x_{k}^{11} \notin B\right) \& \ldots \&\left(x_{1}^{1 n} \notin B \vee \ldots \vee x_{k}^{1 n} \notin B\right)\right) \\
&=\mathbf{P}\left(\left(x_{1}^{11} \notin B\right) \vee \ldots \vee\left(x_{k}^{11} \notin B\right)\right) \ldots \mathbf{P}\left(\left(x_{1}^{1 n} \notin B\right) \vee \ldots \vee\left(x_{k}^{1 n} \notin B\right)\right)
\end{aligned}
$$

В силу закона де Моргана,

$$
\begin{aligned}
& \mathbf{P}\left(\left(x_{1}^{11} \notin B\right) \vee \ldots \vee\left(x_{k}^{11} \notin B\right)\right) \ldots \mathbf{P}\left(\left(x_{1}^{1 n} \notin B\right) \vee \ldots \vee\left(x_{k}^{1 n} \notin B\right)\right) \\
& =\mathbf{P}\left(\overline{\left(x_{1}^{11} \in B\right) \& \ldots \&\left(x_{k}^{11} \in B\right)}\right) \cdots \mathbf{P}\left(\overline{\left(x_{1}^{1 n} \in B\right) \& \ldots \&\left(x_{k}^{1 n} \in B\right)}\right) \\
& =\left(1-\mathbf{P}\left(\left(x_{1}^{11} \in B\right) \& \ldots \&\left(x_{k}^{11} \in B\right)\right)\right) \cdots\left(1-\mathbf{P}\left(\left(x_{1}^{1 n} \in B\right) \& \ldots \&\left(x_{k}^{1 n} \in B\right)\right)\right) \text {. }
\end{aligned}
$$

Заметим, что для всякого $x \in\{k N(k)+1, \ldots, L\}$ векторы $(\underbrace{i, \ldots, i}_{k-1}, x-i(k-1))$, $1 \leqslant i \leqslant\lfloor x / k\rfloor$, попарно не пересекаются. Из (41) и того, что число попарно непересекающихся векторов не меньше $\lfloor x / k\rfloor$ для любого $x \in\{k N(k)+1, \ldots, L\}$, следует справедливость неравенства (38).

Поскольку для любого $x \in\{j L+1, \ldots,(j+1) L-k N(k)-1\}, j=1, \ldots, k-1$, векторы $(\underbrace{L-i, \ldots, L-i}_{k-1}, x-(k-1)(L-i)), 1 \leqslant i \leqslant\lfloor((j+1) L-x) / k\rfloor$, попарно не пересекаются, аналогично (38) получаем, что

$$
\mathbf{P}(x \notin k B) \leqslant\left(\frac{3}{4}\right)^{\lfloor((j+1) L-x) / k\rfloor} .
$$

Теперь докажем справедливость неравенства

$$
\mathbf{P}(P \nsubseteq k B) \leqslant k \sum_{x \geqslant k N(k)+1}\left(\frac{3}{4}\right)^{\lfloor x / k\rfloor} .
$$


Так как

$$
\mathbf{P}(A \vee B) \leqslant \mathbf{P}(A)+\mathbf{P}(B),
$$

получаем, что

$$
\begin{aligned}
\mathbf{P}(P \nsubseteq k B) & =\mathbf{P}(((k N(k)+1) \notin k B) \vee \ldots \vee((k(L-N(k))-1) \notin k B)) \\
& \leqslant \sum_{x=k N(k)+1}^{k L-k N(k)-1} \mathbf{P}(x \notin k B) \\
& =\sum_{x=k N(k)+1} \mathbf{P}(x \notin k B)+\sum_{x=L+1}^{L L-k N(k)-1} \mathbf{P}(x \notin k B)+\ldots+\sum_{x=(k-1) L+1}^{k L-k N(k)-1} \mathbf{P}(x \notin k B) \\
& \leqslant k \sum_{x=k N(k)+1}^{L}\left(\frac{3}{4}\right)^{\lfloor x / k\rfloor} \leqslant k \sum_{x \geqslant k N(k)+1}\left(\frac{3}{4}\right)^{\lfloor x / k\rfloor} .
\end{aligned}
$$

Заметим, что при условии

$$
N(k) \geqslant \frac{\log \left(8 k^{2}\right)}{\log (4 / 3)}
$$

правая часть неравенства (43) не превосходит $1 / 2$. Отсюда следует, что существует по крайней мере $2^{L-k N(k)-1}$ подмножеств $B \subseteq\{1, \ldots, L\}$ таких, что $P \subseteq k B$. Лемма доказана.

Пусть $k \geqslant 2$ - натуральное число и $P \subseteq \mathbf{Z}_{p}-$ произвольная арифметическая прогрессия длины $k(\lfloor p /(2 k-1)\rfloor-1)-(k-1)$. В силу леммы 6,

$$
\left|\mathbf{S S}_{k}\left(\mathbf{Z}_{p}\right)\right| \geqslant\left|\mathbf{S S}_{k}\left(\mathbf{Z}_{p}, P\right)\right| \geqslant \mathbf{C}_{k} 2^{p /(2 k-1)} .
$$

Нижняя оценка теоремы 1 доказана.

Автор выражает признательность своему научному руководителю, профессору А. А. Сапоженко за постановку задачи и внимание к этой работе.

\section{Список литературы}

1. Green B., Ruzsa I., Counting sumsets and sum-free sets modulo a prime. Studia Sci. Math. Hungarica (2004) 41, 285-293.

2. Nathanson M. B., Additive number theory: Inverse problems and the geometry of sumsets. Springer, Berlin, 1996.

3. Pollard J. M., A generalization of the theorem of Cauchy and Davenport. J. London Math. Soc. (1974) 8, 460-462.

4. Сапоженко А. А., Проблема Дедекинда и метод граничных функционалов. Физматлит, Москва, 2009. 